MATHEMATICS OF COMPUTATION

S 0025-5718(99)01057-1

Article electronically published on February 19, 1999

\title{
AN APPROXIMATE INERTIAL MANIFOLDS APPROACH TO POSTPROCESSING THE GALERKIN METHOD FOR THE NAVIER-STOKES EQUATIONS
}

\author{
BOSCO GARCÍA-ARCHILLA, JULIA NOVO, AND EDRISS S. TITI
}

\begin{abstract}
In a recent paper we have introduced a postprocessing procedure for the Galerkin method for dissipative evolution partial differential equations with periodic boundary conditions. The postprocessing technique uses approximate inertial manifolds to approximate the high modes (the small scale components) in the exact solutions in terms of the Galerkin approximations, which in this case play the role of the lower modes (large scale components). This procedure can be seen as a defect-correction technique. But contrary to standard procedures, the correction is computed only when the time evolution is completed. Here we extend these results to more realistic boundary conditions. Specifically, we study in detail the two-dimensional Navier-Stokes equations subject to homogeneous (nonslip) Dirichlet boundary conditions. We also discuss other equations, such as reaction-diffusion systems and the Cahn-Hilliard equations.
\end{abstract}

\section{INTRODUCTION}

The Navier-Stokes (NS) equations (see Section 2), in a smooth bounded domain $\Omega$, with nonslip Dirichlet boundary conditions, can be written as an abstract dissipative evolution equation

$$
\frac{d u}{d t}+\nu A u+R(u)=f
$$

in an appropriate Hilbert space $H$ with norm $|\cdot|$ (see Section 2) (cf. [6], [23], [38]). Here $A: D(A) \subset H \rightarrow H$ is a densely defined, unbounded, self-adjoint and positive operator with compact inverse and contains the higher-order spatial derivatives, $\nu>0$ is a scalar, and $R: D(R) \subset H \rightarrow H$ is a nonlinear map which gathers lower order spatial derivatives and nonlinear terms.

The eigenfunctions $\left\{w_{1}, w_{2}, \ldots\right\}$ of $A$ with the associated eigenvalues $0<\lambda_{1} \leq$ $\lambda_{2}, \ldots$ form a complete orthonormal set in $H$. Let $H_{m}=\operatorname{span}\left\{w_{1}, w_{2}, \ldots, w_{m}\right\}$, $P_{m}$ the orthogonal projection of $H$ onto $H_{m}$ and $Q_{m}=I-P_{m}$. Every solution $u$ of (1) can be decomposed uniquely into

$$
u=p+q, \quad p=P_{m} u, \quad q=Q_{m} u .
$$

The solution $u$ of (1) can be approximated by the Galerkin approximation, $u_{m}(t) \in H_{m}$, obtained by integrating the standard Galerkin method (SGM) which

Received by the editor June 19, 1996 and, in revised form, February 9, 1998.

1991 Mathematics Subject Classification. Primary 65P25.

Key words and phrases. Dissipative equations, spectral methods, approximate inertial manifolds, nonlinear Galerkin methods.

(C)1999 American Mathematical Society 
is given by the system of ordinary differential equations

$$
\frac{d u_{m}}{d t}+\nu A u_{m}+P_{m} R\left(u_{m}\right)=P_{m} f, \quad u_{m}(0)=P_{m} u(0) .
$$

Notice that since $u_{m}$ is sought in $H_{m}$, the Galerkin error $u-u_{m}$ will never be smaller that the best-approximation error $u-p=q$; that is,

$$
\left|u-u_{m}\right| \geq|u-p|=|q| \text {. }
$$

Thus, for a better approximation of $u$, we propose the following algorithm to improve the SGM. We call it the Postprocessing Galerkin Method (PPGM).

Postprocessing Galerkin algorithm. Suppose that the solution $u$ is wanted at time $T>0$. Then,

1. Compute the Galerkin approximation $u_{m}(T)$ by integrating (3) with respect to time.

2. Solve the linear elliptic problem $\nu A q_{m}=Q_{m}\left(f-R\left(u_{m}(T)\right)\right)$.

3. Add $q_{m}$ to $u_{m}(T)$ in order to obtain the new approximation $u_{m}(T)+q_{m}$ to $u(T)$.

We will see that the convergence rate of the new approximation is better than that of the SGM, or, broadly speaking, that $\left|u(T)-\left(u_{m}(T)+q_{m}\right)\right|=o(|q|)$. Moreover, this improvement is obtained at a small computational cost, so that the new method is computationally more efficient than the SGM (see Figure 2 below). We note that in practice, the whole of $q_{m}$ is never computed, and it is replaced by an adequate truncation $P_{m^{\prime}} q_{m}$ with $m^{\prime}>m$ sufficiently large.

The PPGM was introduced and analyzed in [21]. The analysis in [21] relies heavily on the properties of Fourier expansions and the fact that complex exponentials form an algebra; therefore we restricted our treatment in [21] to dissipative evolution PDEs subject to periodic boundary conditions. This may inspire doubts about the PPGM improving the SGM when more realistic boundary conditions are imposed. This question becomes more relevant in view of recent results like those in [4], where, thanks to a clever algorithm for approximating the eigenfunctions and eigenvalues of the Stokes operator $A$, actual computations of (3) are carried out on complex geometries. In the present paper we analyze the method independently of the properties of the eigenfunctions $w_{j}$. We present here a new analysis with much simpler ideas than those presented in [21]. These simpler ideas have allowed us to extend the PPGM to more technical situations [18], [22]. Although for simplicity we concentrate on the Dirichelet (nonslip) boundary conditions and the NS equations, the techniques used here are easily adapted to other situations.

The PPGM is reminiscent of classical defect-correction techniques for steady state problems. To better appreciate this fact, suppose that we are dealing with the steady state problem, so that instead of (1) and (3) we have

$$
\begin{aligned}
\nu A v+R(v) & =f, \quad v \in H, \\
\nu A v_{m}+P_{m} R\left(v_{m}\right) & =P_{m} f, \quad v_{m} \in H_{m},
\end{aligned}
$$

respectively. Thus, in this case the PPGM method would be:

$1^{\prime}$. Find the Galerkin approximation $v_{m} \in H_{m}$ by solving (6).

$2^{\prime}$. Find $z_{m} \in Q_{m} H$ by solving the linear elliptic problem $\nu A z_{m}=$ $Q_{m}\left(f-R\left(v_{m}\right)\right)$.

$3^{\prime}$. Add $z_{m}$ to $v_{m}$ in order to obtain the new approximation $v_{m}+z_{m} \in H$ to $u$. 
Thus, the whole technique amounts to solving in step $1^{\prime}$ the full nonlinear problem in the finite dimensional space $H_{m}$, where nonlinearities are more easily treated, and then computing a correction on its orthogonal complement (or, in practice, an adequate truncation of it) in step $2^{\prime}$. Notice that in step $2^{\prime}$, we just compute the high-frequency component $z_{m}$ of the solution $\tilde{v}=v_{m}+z_{m}$ of the (more familiar) linear elliptic problem

$$
\nu A \tilde{v}=f-R\left(v_{m}\right)
$$

Furthermore, once $\tilde{v}$ is computed, a better approximation $\tilde{\tilde{v}}$ can be computed by replacing $v_{m}$ by $\tilde{v}$ in the nonlinear term $R$ in (7). The process can be further iterated until a prescribed accuracy is reached, or one can use Newton's iteration to converge faster to $v$. Notice also that solving the steady state problem in step $2^{\prime}$ instead of (7) is feasible due to the fact that we are dealing with spectral methods and both $P_{m}$ and $Q_{m}$ commute with $A$. For finite-element methods, one should use (7) instead of step $2^{\prime}$ [11], [22], [43], [44].

Defect-correction techniques, or the related two-grid, two-level or multilevel methods, are a well established technique for nonlinear steady state problems (see e.g. [3], [11], [31], [43], [44], and the references cited therein). That is, the three steps $1^{\prime}-3^{\prime}$ are nothing new. For evolution problems, similar defect-correction techniques are used on the steady state problems that arise when doing implicit time-stepping.

More recently there has been renewed interest in multilevel methods for evolution problems (see e.g. [2], [7], [10], [32], [33], [34]). Part of the recent interest in multilevel methods for evolution problems arose from studies initiated by [14], [16], [13] and [41] in connection with inertial manifolds (IM) [15] and their approximations. In fact, the multilevel methods developed from this approach are not called that, but rather nonlinear Galerkin methods (NLG) [13], [26], [32]. It was through this approach that we arrived at the PPGM.

It may be clarifying then to summarize the main facts of this approach. To do this, notice that, using (2) and the fact that both $P_{m}$ and $Q_{m}$ commute with $A$, equation (1) can be rewritten as the coupled system

$$
\begin{array}{ll}
\frac{d p}{d t}+\nu A p+P_{m} R(p+q)=P_{m} f, & p \in H_{m}, \\
\frac{d q}{d t}+\nu A q+Q_{m} R(p+q)=Q_{m} f, & q \in Q_{m} H .
\end{array}
$$

It is shown in [14] that, under certain circumstances which are motivated by the dynamical systems approach, the $q$ terms other than $\nu A q$ in (9) can be considered negligible as compared to the other terms. Thus, the authors of [14] suggested the following approximation model for the high-frequency component $q$ of $u$ :

$$
q \approx \Phi_{1}(p)=(\nu A)^{-1} Q_{m}(f-R(p)) .
$$

It is noteworthy that in [16] the authors used a totally different technique, which is also motivated by a dynamical systems approach, which led them to a model similar to (10). Notice the similarity of the above approximation with step $2^{\prime}$ of the defectcorrection technique (we will discuss the differences later). In [14] it is also shown that $\Phi_{1}(p)$ is a good approximation to $q$, in the sense that $\left|q-\Phi_{1}(p)\right|=o(|q|)$ (see Section 2 for a more precise statement). Based on this fact, the NLG method was developed. In the NLG method, the exact solution $u$ of (1) is approximated by 
$y_{m}(t)+\Phi_{1}\left(y_{m}(t)\right)$, where $y_{m} \in H_{m}$ is obtained by solving

$$
\begin{gathered}
\frac{d y_{m}}{d t}+\nu A y_{m}+P_{m} R\left(y_{m}+\Phi_{1}\left(y_{m}\right)\right)=P_{m} f, \quad y_{m}(0)=P_{m} u(0), \\
\Phi_{1}\left(y_{m}\right)=(\nu A)^{-1} Q_{m}\left(f-R\left(y_{m}\right)\right),
\end{gathered}
$$

or certain variants of the above equation which preserve the dissipative nature of (1) (cf. [27], [10]).

The NLG error satisfies $\mid u(t)-\left(y_{m}(t)+\Phi_{1}\left(y_{m}(t)\right)|\approx| q-\Phi_{1}(p) \mid=o(|q|)[10]\right.$, which (recall (4)) should imply a better computational performance than the SGM. However, the continuous update of $\Phi_{1}\left(y_{m}\right)$ along the time evolution is so computationally costly that the NLG, in spite of its $o(|q|)$ error, is generally less efficient than the SGM.

Let us clarify this point now with an example from [21]. Consider the 2dimensional NS equations (see Section 2) in the vorticity-streamfunction formulation

$$
\frac{\partial \omega}{\partial t}-\nu \Delta \omega+\nabla \omega \times \nabla \Psi=g
$$

$$
-\Delta \Psi=\omega,
$$

in the spatial domain $\Omega=[0,2 \pi]^{2}$, with $\nu=0.01$ and subject to periodic boundary conditions. Here the vorticity $\omega=(\nabla \times u) \cdot \mathbf{k}$, the velocity $u=\nabla \times(\Psi \mathbf{k})$, the streamfunction is $\Psi$ and $g=\nabla \times f$. We set the forcing term $g$ as $g(x, y, t)=$ $f_{1}(x, y)(2+\cos (t)) / 3$, where

$$
f_{1}(x, y)= \begin{cases}\left(1+\cos \left(4 r_{+}\right)\right)^{2} / 8, & \text { if } r_{+}<\pi / 4 \\ -\left(1+\cos \left(4 r_{-}\right)\right)^{2} / 8, & \text { if } r_{-}<\pi / 4 \\ 0, & \text { otherwise }\end{cases}
$$

$r_{ \pm}=\left|x+i y-\left(\pi(1+i) \pm e^{i \theta} \pi / 2\right)\right|$, and $\theta=0.7$. The function $f_{1}$ represents stirring the fluid in opposite directions at the locations $\pi(1+i) \pm e^{i \theta} \pi / 2$. Let us fix the vorticity $\omega$ at $t=0$ by $\omega(x, y, 0)=\omega_{0}(x, y)$, where $\omega_{0}=(\nu \Delta)^{-1} f_{1}$.

Figures 1 and 2 show the errors of the different methods when they use several values $N$ of Fourier modes in each spatial direction. The results correspond to runs from $t=40 \pi$ to $T=44 \pi$. Results corresponding to the SGM are marked with asterisks and joined by continuous lines, and those of the NLG are marked by small circles and joined by dotted lines. Since in the present example $\omega$ is in the Sobolev spaces $H^{\sigma}$ for all $\sigma<13 / 2$, following suggestions in [41], $\Phi_{1}$ was truncated to $N^{13 / 11}$ Fourier modes in each spatial direction (see also [10], [28] and [37]).

Figure 1 is a convergence diagram where the errors in the vorticity $\omega$ committed by the methods, measured in the $H^{1}$ norm, are plotted versus the number $N$ of Fourier modes in each spatial direction. The slopes of least-squares fits to the last four points of each method are shown in Figure 1. We see that whereas in the SGM the errors decay like $N^{-5.5}$, in the NLG they decay like $N^{-6.5}$. Thus, the NLG has a better convergence rate than the SGM, because its error decays faster with the number of modes ( $m=N^{2}$ in this example) than the SGM.

However, efficiency (ratio of cost to error) is another matter. In Figure 2, the same errors as in Figure 1 are plotted versus the smallest amount of computing time (in seconds) that the methods needed to achieve those errors. It is clear from Figure 2 that the NLG takes more than twice the computing time of the SGM for 


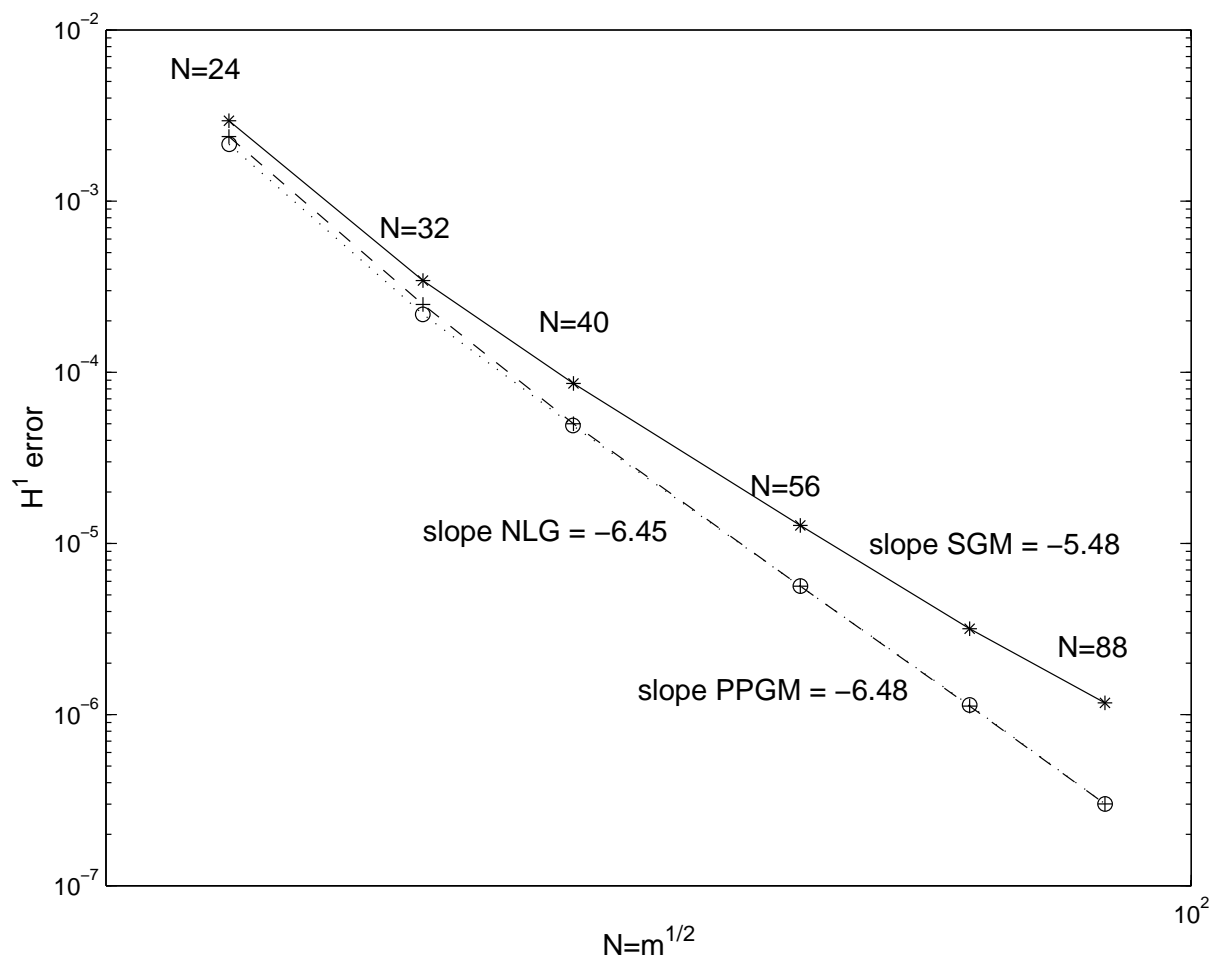

FIgURE 1. Convergence diagram; * SGM, + PPGM, o NLG

any error. This can be seen by drawing any horizontal line (i.e., selecting a desired accuracy); then, its intersection with the continuous line of the SGM is on the right of its intersection with the dotted line of the NLG by a factor of more than two (i.e., to achieve that desired accuracy, the SGM needs less than half the computing time of the NLG).

The poor practical performance of the NLG led us to develop the PPGM. The aim was to obtain the better accuracy of the NLG at the cost of the cheaper SGM. This, as shown in Figures 1 and 2, is achieved by the PPGM, whose results are represented by + sings and joined by dashed lines. In, Figure 1, the NLG and the PPGM commit virtually the same errors for a given $N(\mathrm{o}$ and + signs are plotted on almost the same locations). Furthermore, in Figure 2, the + sings of the PPGM and the $*$ of the SGM are (almost) on the same vertical lines (i.e., same cost) but since the PPGM error is smaller, it turs out to be more efficient. Again, this can be checked by noticing that the broken line joining + sings is the leftmost (i.e., the PPGM needs less computing time to reach a given accuracy).

Two final comments are pertinent in this example. First, following standard practice with spectral Fourier methods, nonlinear terms were approximated by interpolation, in what is known as pseudospectral methods; we made sure that the aliasing error arising from this practice was not dominant (i.e., that the errors were the same as those of the more costly pure spectral methods). Second, we were very careful in checking the sources of error and cost to avoid reaching wrong conclusions 


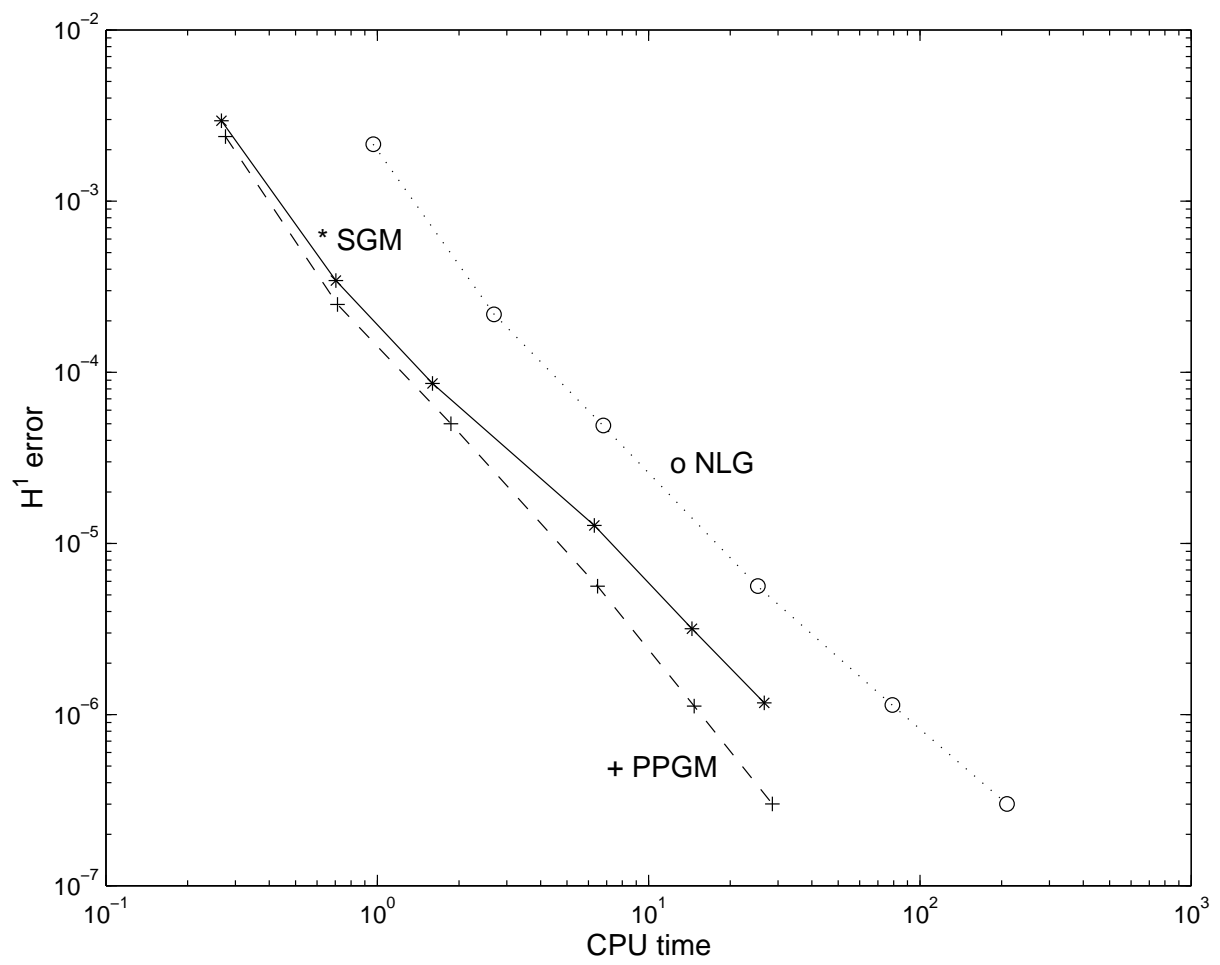

FiguRE 2. Efficiency diagram

on the relative efficiency of the methods (see [19], [20]). We refer the reader to [21], where full details of the methods used are provided, and where $L^{2}$ errors are shown.

Let us turn to the relation of these methods with defect-correction techniques. Notice that both the NLG (11) and the PPGM in steps 1-3 are defect-correction methods for evolution problems: the corrections $\Phi\left(y_{m}\right)$ in the NLG and $q_{m}$ in the PPGM are obtained through solving linear elliptic problems. Notice also that, for the correction, both the NLG and the PPGM use the Foias-Manley-Temam mapping $\Phi_{1}$ in (10), since $q_{m}$ in step 3 is $q_{m}=\Phi_{1}\left(u_{m}(T)\right)$. Let us then look more closely to the relation between $\Phi_{1}$ and the standard defect-correction approach for steady state problems in steps $1^{\prime}-3^{\prime}$. Observe that on going from (9) to (10), the $d q / d t$ term is dropped. Thus, in the steady state case $2,^{\prime}$ we have the full forcing (source) term $f$ of the original problem (5), whereas in the evolution case (10), only $Q_{m} f$ instead of $Q_{m} f-d q / d t$ is present. This implies that while in $1^{\prime}-3^{\prime}$, continuing iteration of the process might bring better and better approximations, this is not (necessarily) so in (10) unless some approximation to $d q / d t$ is included. Procedures for doing this can be found in [8], [9], [10], [39] and [40]. Our practical experience with them [20] inspired us to use only $\Phi_{1}$ in (10). For the possibility of using Newton's or another iteration method see, for instance, [16], [26], [35] and [41].

Observe also that in the PPGM the correction $q_{m}$ is computed only once, as opposed to more standard defect-correction practices for evolution problems like 
the NLG or the method presented in [7], where the correction is computed at every time step over the interval $[0, T]$.

A quick explanation of the $o(|q|)$ error of the PPGM may make things clearer. For linear problems with constant coefficients $u_{m}$ is $p$, so that the error $p-u_{m}=0$. However, $u-u_{m}=q$, which is not negligible (and hence (4)). For nonlinear problems, we show here that $\left|p-u_{m}\right|=o(|q|)$, and this allows $\Phi_{1}\left(u_{m}\right)$ to be a good approximation to $\Phi_{1}(p) \approx q$. In fact, since $\Phi_{1}$ is Lipschitz-continuous (see, e.g., [10]), then $\left|\Phi_{1}\left(u_{m}\right)-\Phi_{1}(p)\right| \leq C\left|u_{m}-p\right|$. Thus, by adding $\left.\pm \Phi_{(} p\right)$, for the error we have that

$$
\left|u_{m}+\Phi_{1}\left(u_{m}\right)-p-q\right| \leq(1+C)\left|u_{m}-p\right|+\left|\Phi_{1}(p)-q\right|=o(|q|) .
$$

We perform the analysis summarized here for both $L^{2}$ and $H^{1}$ norms.

We point out that in [14], the graph of $\Phi_{1}$, that is, $\left\{p+\Phi_{1}(p) \mid p \in H_{m}\right\}$, was called an approximate inertial manifold (AIM) (see also [16], [41]), in reference to the IMs, where $q=\Phi(p)$ for some mapping $\Phi$, which is sometimes known to exist but is hardly ever known explicitly. We do not discuss here whether AIMs approximate IMs or not. We simply use $\Phi_{1}$ and use the term AIM that other authors coined before us.

We remark that our results are not restricted to time-independent forcing terms $f$ in (1), and are valid for Hölder-continuous (in time) $f$ (see Remark 2 in Section 3). We also show how to extend our analysis to dissipative equations other than the NS equations, such as reaction-diffusion systems and the Cahn-Hilliard equations. We note that there are in the literature AIMs other than the $\Phi_{1}$ used here [8], [9], [14], [16], [17], [30], [38], [39], [41]. Our results hold also for the Euler-Galerkin approximate inertial manifold which was introduced in [16] (see also [13] and [26]).

The rest of the paper is organized as follows. In Section 2 some standard preliminary material is introduced. Section 3 is devoted to studying the PPGM for the NS equations. Finally, in Section 4, the extension of the results to other equations is discussed.

\section{Preliminary Results}

We consider the NS equations

$$
\begin{aligned}
u_{t}+(u \cdot \nabla) u+\nabla p & =\nu \Delta u+F, \\
\operatorname{div}(u) & =0,
\end{aligned}
$$

in a smooth bounded domain $\Omega \subset R^{2}$, subject to the homogeneous (nonslip) Dirichlet boundary condition $\left.u\right|_{\partial \Omega}=0$. In the rest of the paper, we use the spaces

$$
H=\left\{u \in L^{2}(\Omega)^{2}|\operatorname{div}(u)=0, u \cdot n|_{\partial \Omega}=0\right\}
$$

and the space

$$
V=\left\{u \in H_{0}^{1}(\Omega)^{2} \mid \operatorname{div}(u)=0\right\} .
$$

Let $\Pi$ be the orthogonal $L^{2}$ projection $\Pi: L^{2}(\Omega)^{2} \rightarrow H$. Projecting onto $H$, the NS equations become equation (1) with $A=-\Pi \Delta, R(u)=B(u, u)=\Pi[(u \cdot \nabla) u]$, and $f=\Pi F$ (see, e.g., [6]). It is well known that then $D\left(A^{1 / 2}\right)=V$. Following the notation of [6], we denote by $(\cdot, \cdot)$ the inner product in $H$ (i.e., in $\left.L^{2}(\Omega)^{2}\right)$, and by $|\cdot|$ and $\|\cdot\|$ the norms

$$
|u|=\|u\|_{L^{2}(\Omega)^{2}}, \quad\|v\|=\left|A^{1 / 2} v\right|
$$


in $H$ and $V$ respectively. We will also denote by $\|\cdot\|_{\infty}$ the $L^{\infty}(\Omega)$ norm. For $k$ an integer, we consider the Sobolev space $H^{k}=H^{k}(\Omega)$ with its usual norm rescaled to

$$
\|u\|_{H^{k}}=\left(\sum_{|\alpha| \leq k} \lambda_{1}^{k-|\alpha|}\left\|D^{\alpha} u\right\|_{L^{2}(\Omega)}^{2}\right)^{1 / 2} .
$$

This norm is readily extended to $H^{k}(\Omega)^{2}$. We will use the inequalities

$$
\|\omega\|_{H^{k}(\Omega)^{2}} \leq c_{1}\left|A^{k / 2} \omega\right|, \quad \forall \omega \in D\left(A^{k / 2}\right), \quad k=1,2,3,
$$

where, throughout this paper, $c, c_{1}, c_{2}, \ldots$ will denote dimensionless constants. (See [6], pp. 36-41, on the regularity requirements on the domain $\Omega$ for (14) to be valid).

We recall the Brézis-Gallouet [5] inequality in two dimensions:

$$
\|u\|_{L^{\infty}(\Omega)} \leq c_{2}\|u\|_{H^{1}}\left(1+\log \frac{\|u\|_{H^{2}}}{\lambda_{1}^{1 / 2}\|u\|_{H^{1}}}\right)^{1 / 2}, \quad \forall u \in H^{2} .
$$

This inequality is also valid for $u \in H^{2}(\Omega)^{2}$, and as a result we have

$$
\|v\|_{\infty} \leq c_{3}\|v\| L_{m}, \quad \forall v \in H_{m}
$$

where

$$
L_{m}=\left(1+\log \frac{\lambda_{m}}{\lambda_{1}}\right)^{1 / 2}
$$

The bilinear form $B$ satisfies the following skew property:

$$
(B(u, v), w)=-(B(u, w), v) \quad \text { for all } \quad u, v, w \in V
$$

(see, for instance, [6], p. 53). Moreover, for any $\delta$ with $1 / 2<\delta<1$ we have

$$
\left|A^{-\delta}(R(u)-R(v))\right| \leq L|u-v|, \quad \forall u, v \in V,
$$

with $L=c_{4}\left|A^{1 / 2}(u+v)\right|$ (see, e.g., [6], p. 55).

We recall the following property of the NS equations. There exist constants $M_{0}$ and $M_{1}$ such that for any solution $u$ of $(1)$, there is a time $T_{0}=T_{0}(|u(0)|,|f|)$ such that

$$
|u(t)| \leq M_{0}, \quad\left|A^{1 / 2} u(t)\right| \leq M_{1}, \quad t \geq T_{0} .
$$

Here and below, $M, M_{0}, M_{1}, \ldots$ and $K, K_{0}, K_{1}, \ldots$ denote constants that may depend on the data of the problem (i.e., $\nu, f,|u(0)|$ and $\left.\lambda_{1}\right)$. A simple modification to the argument given in [14] allows us to choose the time $T_{0}$ large enough so that

$$
\begin{aligned}
|q(t)| & \leq K_{0} \frac{L_{m}}{\lambda_{m+1}}, \quad K_{0}=\frac{c_{5}}{\nu}\left(\frac{\left|Q_{m} f\right|}{L_{m}}+M_{1}^{2}\right), \\
\|q(t)\| & \leq K_{1} \frac{L_{m}}{\lambda_{m+1}^{1 / 2}}, \quad K_{1}=\frac{c_{6}}{\nu}\left(\frac{\left|Q_{m} f\right|}{L_{m}}+M_{1}^{2}+\frac{M_{0} M_{1}^{2}}{\nu L_{m}}\right),
\end{aligned}
$$

for every $m$ and for every $t \geq T_{0}$. In particular these estimates hold, for all $-\infty<$ $t<\infty$, for any solution in the global attractor. We now summarize some results concerning $\Phi_{1}$ and the rates of convergence of the SGM and the NLG. First of all, we notice that the convergence properties of the discretizations of (1) depend on the approximation capabilities of the space where the solution is sought. For the SGM, the solution $u_{m}$ is in $H_{m}$, so that, for example, the $L^{2}$ error cannot be better 
than the $L^{2}$ norm of $u-P_{m} u=u-p=q$. In fact, if (19), (20) and (21) are satisfied for $T_{0}=0$ then

$$
\left|u(t)-u_{m}(t)\right| \leq C(t) \frac{L_{m}}{\lambda_{m+1}}, \quad \text { and } \quad\left\|u(t)-u_{m}(t)\right\| \leq C(t) \frac{L_{m}}{\lambda_{m+1}^{1 / 2}}
$$

(see, for instance, [10] and [36]). Moreover, the above estimate (22) is sharp, as has been demonstrated by an example in [41]. For the NLG, the solution $y_{m}+\Phi_{1}\left(y_{m}\right)$ lies in the manifold $\mathcal{M}_{1}=\operatorname{graph}\left(\Phi_{1}\right)$. The $L^{2}$-distance and the $H^{1}$-distance of the solution $u$ of (1) to this manifold are $\left|q-\Phi_{1}(p)\right|$ and $\left\|q-\Phi_{1}(p)\right\|$, respectively. These quantities can be bounded [14] by

$$
\left|q-\Phi_{1}(p)\right| \leq K_{3} \frac{L_{m}}{\lambda_{m+1}^{3 / 2}}, \quad\left\|q-\Phi_{1}(p)\right\| \leq K_{3} \frac{L_{m}}{\lambda_{m+1}},
$$

with $K_{3}=K_{3}\left(\nu^{-1},|f|, \lambda_{1}, M_{1}^{3}\right)$. One can then prove that

$$
\begin{aligned}
\left|u(t)-y_{m}(t)-\Phi_{1}\left(y_{m}(t)\right)\right| & \leq C(t) \frac{L_{m}^{2}}{\lambda_{m+1}^{3 / 2}}, \\
\left\|u(t)-y_{m}(t)-\Phi_{1}\left(y_{m}(t)\right)\right\| & \leq C(t) \frac{L_{m}^{2}}{\lambda_{m+1}}
\end{aligned}
$$

\section{Main Results}

This section is devoted to proving the following theorem.

Theorem 1. Let $T>0$ be fixed. Let $u$ be a solution of the NS equations on $\left[T_{0}, T\right]$ such that (19), (20) and (21) are satisfied for $T_{0}=0$. Then, there exists a constant $C=C\left(T, M_{1}\right)$ such that for any $t \in(0, T)$ the solution $u_{m}(t)$ of the standard Galerkin method, equation (3), satisfies

$$
\begin{gathered}
\left|p(t)-u_{m}(t)\right| \leq C \frac{L_{m}^{4}}{\lambda_{m+1}^{3 / 2}}, \\
\left|u(t)-\left(u_{m}(t)+\Phi_{1}\left(u_{m}(t)\right)\right)\right| \leq C \frac{L_{m}^{4}}{\lambda_{m+1}^{3 / 2}} . \\
\left\|p(t)-u_{m}(t)\right\| \leq C \frac{L_{m}^{4}}{\lambda_{m+1}}, \\
\left\|u(t)-\left(u_{m}(t)+\Phi_{1}\left(u_{m}(t)\right)\right)\right\| \leq C \frac{L_{m}^{4}}{\lambda_{m+1}} .
\end{gathered}
$$

The factor $L_{m}^{4}$ can be replaced by a constant (see Remark 1 below).

Before proving this theorem, some remarks may be helpful. We first show that, as we mentioned in the Introduction, (27)-(29) are implied by (26). In fact, notice that since $u=p+q$, we can write $u-\left(u_{m}+\Phi_{1}\left(u_{m}\right)\right)=\left(p-u_{m}\right)+\left(q-\Phi_{1}\left(u_{m}\right)\right)$, which, on adding $\pm \Phi_{1}(p)$, can be expressed as

$$
u-\left(u_{m}+\Phi_{1}\left(u_{m}\right)\right)=\left(p-u_{m}\right)+\left(q-\Phi_{1}(p)\right)+\left(\Phi_{1}(p)-\Phi_{1}\left(u_{m}\right)\right) .
$$

The second term on the right hand side above is (recall (23)) $O\left(L_{m} \lambda_{m+1}^{-3 / 2}\right)$. The last term, since $\Phi_{1}$ is Lipschitz-continuous [10], can be bounded as

$$
\left|\Phi_{1}(p)-\Phi_{1}\left(u_{m}\right)\right| \leq l\left|p-u_{m}\right|, \quad\left\|\Phi_{1}(p)-\Phi_{1}\left(u_{m}\right)\right\| \leq l\left\|p-u_{m}\right\|,
$$


where $l$ can be made arbitrary small by choosing $m$ large enough. It is then clear that (27) follows from (26) and (23). Similarly, if we take into account that $\left\|p-u_{m}\right\| \leq \lambda_{m+1}^{1 / 2}\left|p-u_{m}\right|,(28)$ is a straightforward from (26), and by the same argument as above we get $(29)$.

The proof of (26) given below will be divided into a number of separate intermediate results. We now comment on the main ideas, to facilitate the reading. This proof is done by stability plus consistency arguments, reminiscent of finitedifference analysis, rather than by the standard error analysis typical of spectral and finite-element methods. This allows us to study only quantities in $H_{m}$, for example $u_{m}-p$, rather than in quantities $H$ like $u-u_{m}$, and exploit the finite-dimensionality in certain inequalities.

The first step in our proof of (26) is the stability of the SGM. This is no novelty, and is presented in Theorem 2 for convenience of the reader. By stability we mean that we bound the error $\left|p-u_{m}\right|$ in terms of the quantity

$$
W=\max _{0 \leq s \leq T}\left|\int_{0}^{s} e^{-\nu(s-r) A} P_{m} G(r) d r\right|,
$$

which is a convenient norm of the residual $P_{m} G$, where

$$
G=B(p, q)+B(q, p)+B(q, q) .
$$

Notice that $P_{m} G$ equals $(d / d t) p+\nu A p+P_{m} B(p, p)-P_{m} f$; that is, it is the residual or truncation error obtained when $u_{m}$ is replaced by $p$ in the "discrete" equation (3) satisfied by $u_{m}$.

The second step in the proof of (26) is consistency, that is, to show that (32) is of order $L_{m}^{4} \lambda_{m+1}^{-3 / 2}$. Consistency is presented in Theorem 3. From this theorem and the stability of Theorem 2 , the main result of this section, Theorem 1 , follows.

We now comment on the proof of Theorem 3 (consistency), which is prepared by three lemmas. Observe that we want to show that (32) is $O\left(L_{m}^{4} \lambda_{m+1}^{-3 / 2}\right)$. Since $G$ is only of the size of $\|q\|\left(=O\left(\lambda_{m+1}^{-1 / 2}\right)\right)([6])$, p. 50 , in order to get a better rate we must exploit the smoothing effect of $e^{-\nu(s-r) A}$ in (32) by using the inequality

$$
\left|e^{\nu(t-s) A} P_{m} G(s)\right| \leq\left\|A e^{\nu(t-s) P_{m} A}\right\|_{\mathcal{L}\left(H_{m}\right)}\left|A^{-1} P_{m} G(s)\right| .
$$

(Here and in the sequel, $\|\cdot\|_{\mathcal{L}(X)}$ denotes the operator norm in the Hilbert space $X$ ). Lemmas 1 and 2 below show that $\left|A^{-1} P_{m} G(s)\right|=O\left(L_{m}^{2} \lambda_{m+1}^{-3 / 2}\right)$. This result is not valid for $\left|A^{-1} G(s)\right|$, so that the finite-dimensional space $H_{m}$ plays a key role here. After Lemmas 1 and 2, $W$ in (32) can be bounded by

$$
W \leq K L_{m}^{2} \lambda_{m+1}^{-3 / 2} \int_{0}^{T}\left\|A e^{\nu(T-s) P_{m} A}\right\|_{\mathcal{L}\left(H_{m}\right)} d s .
$$

Lemma 3 then shows that $\int_{0}^{t}\left\|A e^{\nu(T-s) P_{m} A}\right\|_{\mathcal{L}\left(H_{m}\right)} d r \leq c L_{m}^{2}$, and consistency follows. Notice that again we have exploited the finite dimension of $H_{m}$, since whereas the operator norm $\left\|A e^{\nu(T-s) P_{m} A}\right\|_{\mathcal{L}\left(H_{m}\right)}$ is integrable in $[0, T]$, the norm $\left\|A e^{\nu(T-s) A}\right\|_{\mathcal{L}(H)}$ is $(\nu(T-s))^{-1}$ and, hence, nonintegrable.

Theorem 2. Let $\delta \in(1 / 2,1)$ be fixed, and let $T>0$ also be fixed. Let $u$ be a solution of the NS equations such that (19), (20) and (21) are satisfied for $T_{0}=0$. 
Then, there exists a constant $C=C\left(T, M_{1}, \delta\right)$ such that for any $t \in[0, T]$ the solution $u_{m}$ of the standard Galerkin method satisfies

$$
\left|p(t)-u_{m}(t)\right| \leq C\left(\left|p(0)-u_{m}(0)\right|+\max _{0 \leq s \leq T}\left|\int_{0}^{s} e^{-\nu(s-r) A} P_{m} G(r) d r\right|\right),
$$

where

$$
p(t)=P_{m} u(t) \quad \text { and } \quad G(r)=R(u(r))-R(p(r)) .
$$

Proof. Let us set $e=p-u_{m}$. Subtracting (3) from (8), we have

$$
\frac{d}{d t} e=-\nu A e+P_{m}\left(R\left(u_{m}\right)-R(p)\right)-P_{m} G .
$$

Hence,

$$
\begin{aligned}
e(t)= & e^{-\nu t A} e(0)+\int_{0}^{t} e^{-\nu(t-s) A} P_{m}\left(R\left(u_{m}(s)\right)-R(p(s))\right) d s \\
& -\int_{0}^{t} e^{-\nu(t-s) A} P_{m} G(s) d s .
\end{aligned}
$$

Taking norms and using (18) and the fact that $\left\|u_{m}(t)\right\| \leq M_{1}$ for $t \geq T_{0}$ (see [6], p. 77), we obtain

$$
|e(t)| \leq\left|e^{-\nu t A} e(0)\right|+\frac{c_{4} M_{1}}{\nu^{\delta}} \int_{0}^{t} \frac{\delta^{\delta}}{(t-s)^{\delta}} L|e(s)| d s+\left|\int_{0}^{t} e^{-\nu(t-s) A} P_{m} G(s) d s\right| .
$$

Now (33) follows from a generalized Gronwall inequality ([24], p. 6).

Lemma 1. There exist a constant c such that for any solution $u$ of the NS equations satisfying (19), (20) and (21) for $T_{0}=0$, the following bounds hold:

$$
\begin{gathered}
\left|A^{-1} P_{m} B(p, q)\right|,\left|A^{-1} P_{m} B(q, p)\right| \leq c M_{1} L_{m}\|q\|_{H^{-1}(\Omega)^{2}}, \\
\left|A^{-1} P_{m} B(q, q)\right| \leq c L_{m}|q|^{2} .
\end{gathered}
$$

Proof. For $u, v \in V$ we have

$$
\begin{aligned}
\left|A^{-1} P_{m} B(u, v)\right| & =\max _{\substack{\xi \in H_{m} \\
|\xi|=1}}\left|\left(A^{-1} B(u, v), \xi\right)\right|=\max _{\substack{\xi \in H_{m} \\
|\xi|=1}}\left|\left(B(u, v), A^{-1} \xi\right)\right| \\
& \left.=\max _{\substack{\xi \in H_{m} \\
|\xi|=1}} \mid\left(B\left(u, A^{-1} \xi\right), v\right)\right) \mid,
\end{aligned}
$$

where for the last equality we have used the skew property (17).

Let us put $\omega=A^{-1} \xi$ (notice that $\omega \in C^{\infty}(\Omega) \cap D(A)$ ). We now replace $u$ and $v$ in (36) by $p$ and $q$ respectively. Since $\operatorname{div}(q)=0$,

$$
(B(p, \omega), q)=(q, p \cdot \nabla \omega) .
$$

Moreover, since $p \in V$ and $\left.\omega \in C^{\infty}(\Omega) \cap D(A)\right)$, then $p \cdot \nabla \omega \in H_{0}^{1}(\Omega)^{2}$, and as a result of the above we have

$$
\begin{aligned}
|(B(p, \omega), q)| & =|(q, p \cdot \nabla \omega)| \leq\|q\|_{H^{-1}(\Omega)^{2}}\|p \cdot \nabla \omega\|_{H_{0}^{1}(\Omega)^{2}} \\
& \leq c\|q\|_{H^{-1}(\Omega)^{2}}\|\nabla(p \cdot \nabla \omega)\|_{L^{2}(\Omega)^{2}} \\
& \leq c\|q\|_{H^{-1}(\Omega)^{2}}\left(\|p\|_{\infty}\|\omega\|_{H^{2}(\Omega)^{2}}+\|\nabla \omega\|_{\infty}\|\nabla p\|_{L^{2}(\Omega)^{2}}\right)
\end{aligned}
$$

Applying the Brézis-Gallouet inequality (15) to $\nabla \omega$, we get

$$
\|\nabla \omega\|_{\infty} \leq c\|\nabla \omega\|_{H^{1}(\Omega)^{2}}\left(1+\log \frac{\|\nabla \omega\|_{H^{2}(\Omega)^{2}}}{\lambda_{1}^{1 / 2}\|\nabla \omega\|_{H^{1}(\Omega)^{2}}}\right)^{1 / 2}
$$


Since $\|\nabla \omega\|_{H^{k}(\Omega)^{2}} \leq\|\omega\|_{H^{k+1}(\Omega)^{2}}$, and using (14) and the fact that $\left|A^{1 / 2} \omega\right|=$ $\|\nabla \omega\|_{L^{2}(\Omega)^{2}}$, we can bound

$$
\frac{\|\nabla \omega\|_{H^{2}(\Omega)^{2}}}{\lambda_{1}^{1 / 2}\|\nabla \omega\|_{H^{1}(\Omega)^{2}}} \leq \frac{\|\nabla \omega\|_{H^{2}(\Omega)^{2}}}{\lambda_{1}\|\nabla \omega\|_{L^{2}(\Omega)^{2}}} \leq \frac{\|\omega\|_{H^{3}(\Omega)^{2}}}{\lambda_{1}\left|A^{1 / 2} \omega\right|} \leq c \frac{\left|A^{3 / 2} \omega\right|}{\lambda_{1}\left|A^{1 / 2} \omega\right|}
$$

Hence, we obtain

$$
\|\nabla \omega\|_{\infty} \leq c|A \omega| L_{m}
$$

Then, from (37), and using (16) to bound $\|p\|_{\infty}$, we get

$$
\left|(B(p, \omega), q)\left\|\leq c^{\prime} L_{m}\right\| p\||A \omega|\| q\right|_{H^{-1}(\Omega)^{2}} .
$$

Since $|A \omega|=1$ and $\|p\| \leq M_{1}$, we conclude that

$$
\left|A^{-1} P_{m} B(p, q)\right| \leq c L_{m} M_{1}\|q\|_{H^{-1}(\Omega)^{2}} .
$$

For $\left|A^{-1} B(q, p)\right|$, arguing as before, we only have to bound $(p, q \cdot \nabla \omega)$. Let $q=\left(q^{1}, q^{2}\right)^{T}$; then we observe that

$$
(p, q \cdot \nabla \omega)=\int_{\Omega} q^{1} p \cdot \partial_{x} \omega d x d y+\int_{\Omega} q^{2} p \cdot \partial_{y} \omega d x d y .
$$

Let us estimate the first term on the right hand side from above. As before, since $p \in V$ and $\omega \in C^{\infty}(\Omega) \cap D(A)$ we have $p \cdot \partial_{x} \omega \in H_{0}^{1}(\Omega)$. Therefore,

$$
\left|\int_{\Omega} q^{1} p \cdot \partial_{x} \omega d x d y\right| \leq c\left\|q^{1}\right\|_{H^{-1}(\Omega)}\left\|\nabla\left(p \cdot \partial_{x} \omega\right)\right\|_{L^{2}(\Omega)^{2}} .
$$

By using arguments similar to the ones above, especially applying the BrézisGallouet inequality (15) twice, and the fact that $p, \omega \in H_{m}$, we obtain

$$
\begin{aligned}
\left\|\nabla\left(p \cdot \partial_{x} \omega\right)\right\|_{L^{2}(\Omega)^{2}} & \leq\|\nabla p\|_{L^{2}(\Omega)^{2}}\left\|\partial_{x} \omega\right\|_{\infty}+\|p\|_{\infty}\left\|\nabla \partial_{x} \omega\right\|_{L^{2}(\Omega)^{2}} \\
& \leq c L_{m}\left(\|p\||A \omega|+\|p\|\|\omega\|_{H^{2}(\Omega)^{2}}\right) \\
& \leq c^{\prime} L_{m}\|p\||A \omega| \leq c^{\prime} L_{m}\|p\|,
\end{aligned}
$$

because $|A \omega|=1$. The term $\int_{\Omega} q^{2} p \cdot \partial_{y} \omega d x d y$ can be treated similarly, and we conclude that

$$
\left|A^{-1} P_{m} B(q, p)\right| \leq c L_{m}\|p\|\|q\|_{H^{-1}(\Omega)^{2}},
$$

which, together with (38), readily leads to (34), since $\|p\| \leq M_{1}$.

To prove (35), let us apply (36) with $u=v=q$ and use (17) to obtain

$$
\left.\left|A^{-1} P_{m} B(q, q)\right| \leq \max _{\substack{\xi \in H_{m} \\|\xi|=1}} \mid\left(B\left(q, A^{-1} \xi\right), q\right)\right)\left.|\leq| q\right|^{2} \max _{\substack{\xi \in H_{m} \\|\xi|=1}}\left\|\nabla A^{-1} \xi\right\|_{\infty} \leq c L_{m}|q|^{2},
$$

where in the last step we have applied the Brézis-Gallouet inequality (15) to $\left\|\nabla A^{-1} \xi\right\|_{\infty}$ and used the fact that $\xi \in H_{m}$.

Lemma 2. There exist a constant $c>0$ such that for any $q \in V$ the following bound holds:

$$
\|q\|_{H^{-1}(\Omega)^{2}} \leq c\left|A^{-1 / 2} q\right| .
$$


Proof. Notice that $\|q\|_{H^{-1}(\Omega)^{2}}=\sup \left\{|(q, v)| \mid v \in H_{0}^{1}(\Omega)^{2},\|v\|_{H_{0}^{1}(\Omega)^{2}}=1\right\}$. But

$$
\begin{aligned}
|(q, v)| & =|(q, \Pi v)|=\left|\left(A A^{-1} q, \Pi v\right)\right|=\left|\left(\Delta A^{-1} q, \Pi v\right)\right|=\left|\left(\nabla A^{-1} q, \nabla \Pi v\right)\right| \\
& \leq\left|\nabla A^{-1} q\right||\nabla \Pi v|=\left|A^{1 / 2} A^{-1} q\right||\nabla \Pi v|=\left|A^{-1 / 2} q\right||\nabla \Pi v|,
\end{aligned}
$$

where we recall that $\Pi$ is the orthogonal projection of $L^{2}$ onto $H$. Since (see Remark 1.10 in [6], p. 9) $\Pi: H_{0}^{1} \rightarrow H^{1}$ is continuous, (39) follows.

Lemma 3. Let $g:[0, T] \rightarrow H_{m}$ be such that

$$
\max _{0 \leq t \leq T}\left|A^{-1} g(t)\right| \leq C \text {. }
$$

Then for any $t \in[0, T]$,

$$
\left|\int_{0}^{t} e^{-(t-s) \nu A} g(s) d s\right| \leq \frac{L_{m}^{2}}{\nu} C .
$$

Proof. We start by noticing that

$$
\int_{0}^{t} e^{-(t-s) \nu A} g(s) d s=\frac{1}{\nu} \int_{0}^{t} \nu A e^{-(t-s) \nu A} A^{-1} g(s) d s .
$$

Since $g(s) \in H_{m}$ for $s \in[0, T]$, we have

$$
A e^{-(t-s) \nu A} A^{-1} g(s)=P_{m} A e^{-(t-s) \nu P_{m} A} A^{-1} g(s),
$$

so that

$$
\left|\int_{0}^{t} e^{-(t-s) \nu A} g(s) d s\right| \leq C \frac{1}{\nu} \int_{0}^{t}\left\|\nu P_{m} A e^{-(t-s) \nu P_{m} A}\right\|_{\mathcal{L}\left(H_{m}\right)} d s .
$$

In the sequel, we change variables $r=t-s$ in the integral above. Observe that

$$
\begin{aligned}
\left\|\nu P_{m} A e^{-r \nu P_{m} A}\right\|_{\mathcal{L}\left(H_{m}\right)} & =\max _{1 \leq j \leq m} \nu \lambda_{j} e^{-\nu \lambda_{j} r} \leq \max _{z \in\left[\nu \lambda_{1}, \nu \lambda_{m}\right]} z e^{-r z} \\
& = \begin{cases}\nu \lambda_{m} e^{-\nu \lambda_{m} r} & \left(r \leq 1 /\left(\nu \lambda_{m}\right)\right), \\
\frac{e^{-1}}{r} & \left(1 /\left(\nu \lambda_{m}\right) \leq r \leq 1 /\left(\nu \lambda_{1}\right)\right), \\
\nu \lambda_{1} e^{-\nu \lambda_{1} r} & \left(r \geq 1 /\left(\nu \lambda_{1}\right)\right) .\end{cases}
\end{aligned}
$$

In order to estimate the right hand side of (41) we decompose the interval $[0, t]$ as the union of

$$
I_{1}=\left[0, \frac{1}{\nu \lambda_{m}}\right] \cap[0, t], \quad I_{2}=\left[\frac{1}{\nu \lambda_{m}}, \frac{1}{\nu \lambda_{1}}\right] \cap[0, t], \quad I_{3}=\left[\frac{1}{\nu \lambda_{1}}, t\right] \cap[0, t] .
$$

We then have

$$
\begin{gathered}
\int_{I_{1}}\left\|\nu P_{m} A e^{-r \nu P_{m} A}\right\|_{\mathcal{L}\left(H_{m}\right)} d r \leq \int_{I_{1}} \nu \lambda_{m} e^{-\nu \lambda_{m} r} d r \leq 1-\frac{1}{e} \\
\int_{I_{2}}\left\|\nu P_{m} A e^{-r \nu P_{m} A}\right\|_{\mathcal{L}\left(H_{m}\right)} d r \leq \frac{1}{e} \int_{I_{2}} \frac{1}{r} d r \leq \frac{L_{m}^{2}}{e}
\end{gathered}
$$

and

$$
\int_{I_{3}}\left\|\nu P_{m} A e^{-r \nu P_{m} A}\right\|_{\mathcal{L}\left(H_{m}\right)} d r \leq \int_{I_{3}} \nu \lambda_{1} e^{-\nu \lambda_{1} r} d r \leq \frac{1}{e}-e^{-\lambda_{1} t} .
$$

Combine the above with (41) to obtain (40). 
Theorem 3. Suppose that the conditions of Theorem 2 hold. Then there exists a constant $c>0$ such that

$$
\begin{aligned}
\max _{t \geq 0}\left|\int_{0}^{t} e^{-\nu(t-s) A} P_{m} G(s) d s\right| & \leq \frac{L_{m}^{2}}{\nu}\left(L_{m} M_{1}\left|A^{-1 / 2} q\right|+c L_{m}|q|^{2}\right) \\
& \leq \frac{K_{0} L_{m}^{4}}{\nu \lambda_{m+1}^{3 / 2}}\left(M_{1}+\frac{c L_{m} K_{0}}{\lambda_{m+1}^{1 / 2}}\right) .
\end{aligned}
$$

Proof. Let us recall that

$$
P_{m} G=P_{m} B(p, q)+P_{m} B(q, p)+P_{m} B(q, q) .
$$

Then (42) follows immediately from (20), (21), and Lemmas 1, 2 and 3.

Remark 1 . The leading term $\nu^{-1} L_{m}^{4} K_{0} M_{1} \lambda_{m+1}^{-3 / 2}$ on the right hand side of (42) can be replaced by $\nu^{-2} L_{m}^{2} M_{1}^{3} K_{0} \lambda_{m+1}^{-3 / 2}$ in the following way. Let us denote $g(s)=$ $B(p, q)+B(q, p)$. Using integration by parts we can write

$$
\begin{aligned}
\int_{0}^{t} e^{-\nu(t-s) A} P_{m} g(s) d s= & \frac{1}{\nu}\left(A^{-1} P_{m} g(t)-e^{-\nu t A} A^{-1} P_{m} g(0)\right) \\
& -\frac{1}{\nu} \int_{0}^{t} e^{-\nu(t-s) A} A^{-1} P_{m} \frac{d g(s)}{d s} d s .
\end{aligned}
$$

Notice that no term $A e^{\nu(t-s) A}$ appears now, so that the $L_{m}^{2}$ factor arising from Lemma 3 will not be present in the corresponding bound. Then the result follows from the the fact that for $t \geq T_{0}$,

$$
\left\|\frac{d u}{d t}(t)\right\| \leq c \frac{M_{1}^{2}}{\nu} M_{1}, \quad\left|\frac{d q}{d t}(t)\right| \leq c \frac{M_{1}^{2}}{\nu} K_{0} \frac{L_{m}}{\lambda_{m+1}} .
$$

(Here, we are assuming that $|f|$ is not too small; see [14] for a more precise statement of (44)). Notice that the above estimate (44) holds for all $t \in(-\infty, \infty)$ for solutions in the global attractor. Let us remark, however, that this approach provides a more favorable estimate than (42) only if $m$ is sufficiently large so that $L_{m}>M_{1}^{2} / \nu$.

Furthermore, all terms $L_{m}$ can be removed from (42) at the price of replacing all constants depending on $M_{1}$ by constants depending on $M_{2}=\max _{t \geq T_{0}}|A u|$, which is several orders of magnitude bigger than $M_{1}$.

Remark 2. Theorem 1 is not restricted to time-independent forcing terms. If $f=$ $f(t)$ satisfies $f_{\infty}=\max _{t \geq 0}|f(t)|<+\infty$ and for some $\theta \geq 1 / 2$

$$
\left|f\left(t_{1}\right)-f\left(t_{2}\right)\right| \leq L_{f}\left|t_{1}-t_{2}\right|^{\theta}, \quad \forall t_{1}, t_{2} \geq 0,
$$

then it can be shown (see [29]) that (23) holds (inequality (23) was first proved in [14] for time-independent forces $f$ ). Since $f$ plays no role in the results in this section, they are also valid for $f=f(t)$.

It is important to notice that for time-dependent forces, the estimates (44) do not necessarily hold (except for $f$ analytic in a strip of the complex plane around the positive real time axis). Hence the approach provided by (43) is of no use, and Lemma 3 has to be used. 


\section{Further EXTEnsions}

The fact that the postprocessed Galerkin method has the same rate of convergence as the NLG with $\Phi_{a p p}=\Phi_{1}$ is not an exclusive property of the NS equations. It applies also to other two-dimensional (and three-dimensional) dissipative PDEs. In fact, none of the properties of the solutions of the NS equations that have played a key role in our analysis are exclusive to the NS equations, nor are the three lemmas in the previous section applicable only to the NS equations.

Let us review first the properties of the NS equations that we have used. These are (19) (i.e., dissipativity) and (14) (i.e., the continuity of the embeddings of $D\left(A^{\alpha}\right)$ into the corresponding Sobolev spaces). Notice that we have used (14) in order to have some control on the $L^{\infty}$ norms of both $p$ and $u_{m}$ (and their spatial derivatives) in the nonlinear terms. To avoid the presence of $|A u|$ in our error bounds we relied heavily on the Brézis-Gallouet inequality (15). This works nicely for twodimensional problems; however, for three-dimensional problems we will have to use the estimate

$$
M_{2}=\max _{t \geq T_{0}}|A u|
$$

instead.

As for the lemmas in the previous section, Lemma 3 is valid even for sectorial operators, while Lemma 2 depends on the boundary conditions and the relation between Sobolev spaces and the the fractional powers of $A$. Lemma 1, however, depends on the particular nonlinearity of the equation.

We show now how to extend the results of the previous section to reactiondiffusion (RD) equations of the form

$$
u_{t}-\nu \Delta u+R(u)=f,
$$

where $R$ is a polynomial nonlinearity

$$
R(u)=\sum_{j=1}^{2 k+1} a_{j} u^{j}, \quad a_{2 k+1}>0 .
$$

We consider the equation on a bounded domain $\Omega$ in $R^{d}, d \leq 3$, subject to homogeneous Dirichlet boundary conditions $u=0$ on $\partial \Omega$. Notice that the operator $A$ is $A=-\Delta$. We refer the reader to, for instance, [38] for the properties of the solutions of this equation.

For the RD equation (46), the mapping $\Phi_{1}$ possesses the following approximation property [8]. For $u=p+q$ satisfying (19) and (45)

$$
\left|q-\Phi_{1}(p)\right| \leq \frac{K_{4}}{\lambda_{m+1}^{2}},
$$

where $K_{4}$ depends on $M_{2}$ (in fact, on $M_{2}^{2 k}$ ). All the bounds that we obtain here for the RD equation will depend on this constant $M_{2}$, rather than on $M_{1}$.

We present a version of Lemma 1 for the RD equation.

Lemma 4. There exists a constant $c$ such that for any solution $u$ of the $R D$ equation (46) such that (19) and (45) are satisfied for $T_{0} \geq 0$, the following bound holds:

$$
\left|A^{-1}(R(u)-R(p))\right| \leq K M_{1}^{k-1 / 2} M_{2}^{k+1 / 2}\left|A^{-1} q\right| .
$$


Proof. Reasoning as in Lemma 1, we have to bound $\left|\left(R(u)-R(p), A^{-1} \xi\right)\right|$ with $|\xi|=1$. Notice that $R(u)-R(p)$ is a linear combination of terms that are either $q$, or $q^{l} p^{n}$ with $l \geq 1$ and $2 \leq l+n \leq 2 k+1$. Then for $\omega=A^{-1} \xi$ we denote $v=q^{l-1} p^{n} \omega$ and $Q=A^{-1} q$. Since $D(A)$ is an algebra, and since $q, p$ and $\omega$ belong to $D(A)$, then $\nabla v=0$ on $\partial \Omega$. As a result we have

$$
\left(q^{l} p^{n}, \omega\right)=(q, v)=-(\operatorname{div}(\nabla Q), v)=(\nabla Q, \nabla v)=-(Q, \Delta v),
$$

where, for the last equality, we have used the fact that $Q \nabla v=0$ on $\partial \Omega$. Then, if we show that $|\Delta v| \leq K M_{1}^{k-1 / 2} M_{2}^{k+1 / 2}|\xi|$, (49) will follow. Assume for simplicity that $l=1$ (the case $l \geq 1$ is treated similarly). Then

$$
\Delta v=n \omega p^{n-1} \Delta p+n(n-1) \omega p^{n-2} \nabla p \cdot \nabla p+2 n p^{n-1} \nabla p \cdot \nabla \omega+p^{n} \Delta \omega .
$$

We bound each term on the right hand side above separately. Take the first term. Using Agmon's inequality [1], [38] in three dimensions

$$
\|f\|_{\infty} \leq c\|f\|_{H^{1}}^{1 / 2}\|f\|_{H^{2}}^{1 / 2}, \quad \forall f \in H^{2}(\Omega)
$$

and (13), we have

$$
\begin{aligned}
\left|\omega p^{n-1} \Delta p\right| & \leq\|\omega\|_{\infty}\|p\|_{\infty}^{n-1}|\Delta p| \leq c M_{1}^{\frac{n-1}{2}} M_{2}^{\frac{n+1}{2}}\|\omega\|_{H^{1}}^{1 / 2}\|\omega\|_{H^{2}}^{1 / 2} \\
& \leq c \lambda_{1}^{-1 / 4} M_{1}^{k-1 / 2} M_{2}^{k+1 / 2}|\xi|
\end{aligned}
$$

where for the last inequality we have used the fact that $\|\omega\|_{H^{1}} \leq \lambda_{1}^{-1 / 2}\|\omega\|_{H^{2}}$. For the first term on the right hand side of (50) we write

$$
\begin{aligned}
\left|p^{n} \Delta \omega\right| & \leq\|p\|_{\infty}^{n}|\Delta \omega| \leq c\|p\|_{H^{1}}^{n / 2}\|p\|_{H^{2}}^{n / 2}|\xi| \leq c\|p\|_{H^{1}}^{1 / 2} M_{1}^{\frac{n-1}{2}} M_{2}^{n / 2}|\xi| \\
& \leq c \lambda_{1}^{-1 / 4}\|p\|_{H^{2}}^{1 / 2} M_{1}^{\frac{n-1}{2}} M_{2}^{n / 2}|\xi| \leq c \lambda_{1}^{-1 / 4} M_{1}^{k-1 / 2} M_{2}^{k+1 / 2}|\xi| .
\end{aligned}
$$

For the third term on the right hand side of (50) we first notice that, using Hölder's inequality and Sobolev's inequalities in three dimensions, we have the bound

$$
\begin{aligned}
|\nabla p \cdot \nabla \omega| & \leq\|\nabla p\|_{L^{3}(\Omega)^{2}}\|\nabla \omega\|_{L^{6}(\Omega)^{2}} \leq c\|\nabla p\|_{H^{1 / 2}(\Omega)^{2}}\|\nabla \omega\|_{H^{1}(\Omega)^{2}} \\
& \leq c \lambda_{1}^{-1 / 4}\|p\|_{H^{2}}\|\omega\|_{H^{2}},
\end{aligned}
$$

so that

$$
\begin{aligned}
\left|p^{n-1} \nabla p \cdot \nabla \omega\right| & \leq\|p\|_{\infty}^{n-1}|\nabla p \cdot \nabla \omega| \leq c \lambda_{1}^{-1 / 4} M_{2}^{\frac{n+1}{2}} M_{1}^{\frac{n-1}{2}}|\xi| \\
& \leq c \lambda_{1}^{-1 / 4} M_{1}^{k-1 / 2} M_{2}^{k+1 / 2}|\xi| .
\end{aligned}
$$

Since the second term on the right hand side of (50) can be treated similarly, the proof is complete.

After this lemma, if we want to show that, for the $\mathrm{RD}$ equation, the solution $u_{m}$ of the SGM satisfies $\left|p-u_{m}\right|=O\left(L_{m}^{2} / \lambda_{m+1}^{2}\right)$, we can proceed exactly as in the case of the two-dimensional NS equations. For the three-dimensional case, one always has to replace the Brézis-Gallouet inequality (16) by Agmon's inequality (51).

Remark 3. The results on the RD equation can be also extended with minor modifications to the Cahn-Hilliard equation

$$
u_{t}+\nu \Delta^{2} u+\Delta R(u)=f
$$


$(R$ as in (47)) with boundary conditions

$$
\partial_{n} u=\partial_{n} \Delta u=0,
$$

where $\partial_{n}$ denotes normal derivative (see [38]). If $A=-\Delta$, then $\left|p-u_{m}\right|=$ $O\left(L_{m}^{2} / \lambda_{m+1}^{3}\right)$; that is, the same order of convergence (except for the $L_{m}^{2}$ term) as the approximation order of $\Phi_{1}[8]$.

\section{ACKNOWLEDGMENTS}

This research was supported in part by the National Science Foundation and DGICYT projects PB92-0254 and PB95-0216. EST would like to thank the Isaac Newton Institute for the its hospitality where this work was initiated. This work was completed while EST was the Orson Anderson Visiting Scholar at the Institute for Geophysics and Planetary Physics (IGPP) at the Los Alamos National Laboratory.

\section{REFERENCES}

[1] S. Agmon, Lectures on Elliptic Boundary Value Problems. Mathematical Studies, Van Nostrand, Princeton, NJ, 1965. MR 31:2504

[2] A. Ait Ou Ammi and M. Marion, Nonlinear Galerkin methods and mixed finite elements: Two -grid algorithms for the Navier-Stokes equations, Numer. Math., 68, 1994, 189-213. MR 95c:65174

[3] R. E. Bank and D. J. Rose, Analysis of multilevel iterative methods for nonlinear finite element equations, Math. Comp., 39 , 1982, 453-465. MR 83j:65105

[4] P. F. Batcho and G. E. Karniadakis, Generalized Stokes Eigenfunctions: a new trial basis for the solution of incompressible Navier-Stokes equations, J. Comp. Phys., 115, 1994, 121-146. MR 95g:76037

[5] H. Brézis and T. Gallouet, Nonlinear Schrödinger evolution equations, Nonlin. Anal., 4, 1980, 677-681. MR 81i:35139

[6] P. Constantin and C. Foias, Navier-Stokes Equations, Chicago Lectures in Mathematics, The University of Chicago, 1988. MR 90b:35190

[7] C. N. Dawson and M. F. Wheeler, Two-Grid methods for mixed Finite Element approximations of nonlinear parabolic equations, Contemporary Mathematics, 180, 1994 192-204. MR 95j:65117

[8] A. Debussche and M. Marion, On the construction of families of approximate inertial manifolds, J. Diff. Eq., 100, 1992, 173-201. MR 94e:35076

[9] C. Devulder and M. Marion, A class of numerical algorithms for large time integration: the nonlinear Galerkin method, SIAM J. Numer. Anal., 29, 1992, 462-483. MR 93d:65079

[10] C. Devulder, M. Marion and E. S. Titi, On the rate of convergence of Nonlinear Galerkin methods, Math. Comp., 60, 1993, 495-514. MR 93h:76017

[11] J. Douglas, Jr. and T. Dupont, A Galerkin method for a nonlinear Dirichlet problem, Math. Comp., 29, 1975, 698-696. MR 55:4742

[12] T. Dubois, F. Jauberteau, M. Marion and R. Temam, Subgrid modelling and interaction of small and Large wavelengths in turbulent flows, Comp. Phys. Comm., 65, 1991, 100-106. MR 92b: 35123

[13] C. Foias, M. S. Jolly, I. G. Kevrekidis, G. R. Sell and E. S. Titi, On the computation of inertial manifolds, Phys. Lett. A, 131, 1988, 433-436. MR 89k:65154

[14] C. Foias, O. Manley and R. Temam, Modelling of the interaction of small and large eddies in two dimensional turbulent flows, RAIRO Math. Mod. Num. Anal., 22, 1988, 93-114. MR 89h:76022

[15] C. Foias, G. R. Sell and R. Temam, Inertial manifolds for nonlinear evolutionary equations, J. Diff. Eq., 73, 1988, 309-353. MR 89e:58020

[16] C. Foias, G. R. Sell and E. S. Titi, Exponential tracking and approximation of inertial manifolds for dissipative nonlinear equations, J. Dynamics and Diff. Eq., 1, 1989, 199-243. MR 90k:35031

[17] C. Foias, R. Temam, Algebraic approximation of attractors: the finite dimensional case, Physica D, 32, 1988, 163-182. MR 89k:58161 
[18] J. de Frutos, B. García-Archilla and J. Novo, A postprocessed Galerkin method with Chebyshev and Legendre polynomials, (submitted).

[19] B. García-Archilla, Some practical experience with the time integration of dissipative equations J. Comp. Phys., 122, 1995, 25-29. CMP 96:03

[20] B. García-Archilla and J. de Frutos, Time integration of the nonlinear Galerkin method, IMA J. Num. Anal., 14, 1994, 221-244. MR 96h:65121

[21] B. García-Archilla, J. Novo and E. S. Titi, Postprocessing the Galerkin method: a novel approach to approximate inertial manifolds, SIAM J. Numer. Anal. 35, 1998, 941-972. CMP 98:11

[22] B. García-Archilla and E. S. Titi, Postprocessing Galerkin methods: The finite-element case, (submitted)

[23] J. Hale, Asymptotic Behavior of Dissipative Systems. Mathematical Surveys and Monographs, 25, AMS, Providence, 1988. MR 89g:58059

[24] D. Henry, Geometric Theory of Semilinear Parabolic Equations. Springer-Verlag, Berlin, 1988. MR 83j:35084

[25] F. Jauberteau, C. Rosier and R. Temam, A nonlinear Galerkin method for the Navier-Stokes equations, Comput. Meth. Appl. Mech. Eng., 8, 1990, 245-260. MR 91k:76130

[26] M. S. Jolly, I. G. Kevrekidis, E. S. Titi, Approximate inertial manifolds for the KuramotoSivashinsky equation: analysis and computations, Physica D, 44, 1990, 38-60. MR 91f:35217

[27] M. S. Jolly, I. G. Kevrekidis, E. S. Titi, Preserving dissipation in approximate inertial forms for the Kuramoto-Sivashinsky equation, Jour. of Dynamics and Diff. Eq. , 3, 1991, 179-197. MR 92b:35165

[28] D. A. Jones, L. G. Margolin and E. S. Titi, On the effectiveness of the approximate inertial manifold - A computational study, Theor. Comp. Fluid Dynam., 7, 1995, 243-260.

[29] D. A. Jones and E. S. Titi, A remark on quasi-stationary approximate inertial manifolds for the 2D Navier-Stokes equations, SIAM J. Math. Anal., 25, 1994, 894-914. MR 95c:35173

[30] D. A. Jones and E. S. Titi, $\mathrm{C}^{1}$ approximations of inertial manifolds for dissipative nonlinear equations, Jour. Diff. Equ., 127, 1996, 54-86. MR 97g:58154

[31] L. Mansfield, On the solution of nonlinear finite element systems, SIAM J. Numer. Anal., 17, 1980, 752-765. MR 82a:65090

[32] M. Marion and R. Temam, Nonlinear Galerkin Methods, SIAM J. Numer. Anal., 26, 1989, 1139-1157. MR 91a:65220

[33] M. Marion and R. Temam, Nonlinear Galerkin methods: The finite element case, Numer. Math., 57, 1990, 205-226. MR 92d:65200

[34] M. Marion and J. Xu, Error estimates on a new nonlinear Galerkin method based on two-grid finite elements, SIAM J. Numer. Anal., 32, 1995, 1170-1184. MR 96f:65136

[35] J. Novo, Postproceso de Métodos espectrales. Ph. D. Thesis, Universidad de Valladolid, 1997.

[36] R. Rautmann, On the convergence rate of nonstationary Navier-Stokes approximations, Proceedings of IUTAM Sympos., Paderborn (Germany) 1979, Lecture Notes in Mathematics, 771, Springer -Verlag, Berlin, New York, 1980, 425-449. MR 81c:35106

[37] J. Shen, Long time stability and convergence for fully discrete nonlinear Galerkin methods, Appl. Anal., 38, 1990, 201-229. MR 93a:65130

[38] R. Temam, Infinite Dimensional Dynamical Systems in Mechanics and Physics, Applied Mathematical Sciences, 68, Springer-Verlag, Berlin, 1988. MR 89m:58056

[39] R. Temam, Attractors for the Navier-Stokes equations: localization and approximation, $J$. Fac. Sci., Univ. of Tokyo, Sec. IA Math., 36, 1989, 629-647. MR 90m:58128

[40] R. Temam, Dynamical systems, turbulence and the numerical solution of the Navier Stokes equations, in Proceedings of the 11th International Conference on Numerical Methods in Fluid Dynamics (Williamsburg), eds. D. Dwoyer and R. Voigt, Lecture Notes in Physics, Springer-Verlag, Berlin, 1990, pp. 78-98. MR 90i:76055

[41] E. S. Titi, On approximate inertial manifolds to the Navier-Stokes equations, J. Math. Anal. Appl., 149, 1990, 540-557. MR 91j:35226

[42] R. Wallace and D. Sloan, Numerical solutions of a nonlinear dissipative system using a pseudospectral method and inertial manifolds, SIAM J. Sci. Comput., 16, 1995, 1049-1070. MR 97b:65136

[43] J. Xu, A novel two-grid method for semi-linear elliptic equations, SIAM J. Sci. Comput., 15, 1994, 231-237. MR 94m:65178 
[44] J. Xu, Two-grid discretization techniques for linear and nonlinear problems, SIAM J. Numer. Anal., 33, 1996, 1759-1777. MR 97i:65169

Departamento de Matemáticas, Universidad Autónoma de Madrid, 28049 Madrid, SPAIN

E-mail address: bosco.garcia@uam.es

Departamento de Matemática Aplicada y Computación, Universidad de Valladolid, VALLADOLID, Spain

E-mail address: jnovo@mac.mac.cie.uva.es

Department of Mathematics and Department of Mechanical and Aerospace EngiNeEring, University of CALifornia, Irvine, CA 92697-3875, USA

E-mail address: etiti@math.uci-edu 